with cerebrovascular accident, 4/63 (6\%) with bowel infarction, 14/63 (22\%) with avascular necrosis and a single patient with pulmonary infarction (Figure 1). Patients with CVD had a higher disease duration at time of death compared to patients without CVD $(12 \pm 8$ vs. $7 \pm 6$ years), as well as higher cumulative proportions of hematologic disorder (60/63 vs. $15 / 27)$, lymphopenia (48/63 vs. $10 / 27)$, pulmonary damage (19/63 vs. $1 / 27)$, fractures (25/63 vs. $2 / 27)$, higher overall damage $(6.0 \pm 3.0$ vs. $2.4 \pm 2.0)$ and a higher proportion of secondary antiphospholipid syndrome ( $14 / 63$ vs. $1 / 27)(p<0.05)$. Conversely, patients with CVD had a lower proportion of discoid lupus at diagnosis (7/49 vs. 9/24) and a lower proportion of skin damage one year following diagnosis (2/63 vs. $5 / 27)$ $(p<0.05)$. Parameters associated with cardiovascular damage in the multivariate model were cumulative fulfillment of lymphopenia as a classification criterion (odds ratio, OR 4.7 (95\% confidence interval, $\mathrm{Cl} 1.3-17.0)$ ) and accrual of pulmonary damage (OR $13.1(95 \% \mathrm{Cl} 2.2-76.3)$ ).

Figure 1. Distribution of cardiovascular damage in the analyzed group (numbers represent frequencies of each subtype of cardiovascular damage)

$\begin{gathered}\text { Pulmonary } \\ \text { infarction }\end{gathered}$
$\begin{gathered}\text { Avascular } \\ \text { necrosis }\end{gathered}$

Bowel infarction

Conclusions: More than two thirds of deceased patients accrued CVD over the disease course. Lymphopenia and pulmonary damage may be associated with CVD in deceased SLE patients.

References:

[1] Vila LM et al. Arthritis Rheum 2006;55:799-806.

[2] Becker-Merok A and Nossent JC. Lupus 2009;18:508-15.

Disclosure of Interest: None declared

DOI: 10.1136/annrheumdis-2017-eular.5197

\section{FRI0283 AN IMMUNOLOGICAL PROFILE COMBINING INNATE AND ADAPTATIVE IMMUNITY BIOMARKERS IDENTIFY RISK FOR EVOLUTION INTO SLE IN WOMEN WITH RECURRENT PREGNANCY LOSS}

J. Carbone, E. Sarmiento, J.P. Navarro, E. Fernandez-Cruz. Clinical Immunology, Hospital General Universitario Gregorio Marañon, Madrid, Spain

Background: Autoantibodies, low complement levels and higher NK cell counts are present in a subset of women with recurrent pregnancy loss (RPL). The combination of these abnormalities might be a surrogate profile for the presence of a subclinical inflammatory or autoimmune condition.

Objectives: In a cohort of women with unexplained RPL we evaluated if an immunological profile combining innate and adaptive immunity mediators was associated with the presence of distinct clinical characteristics that are commonly observed in autoimmune diseases and if it was a risk factor for developing these diseases. In a small subset of women with the immunological profile we evaluated the activation status of CD4+ and CD8+ cells.

Methods: We evaluated 366 women with RPL defined as 2 or more pregnancy losses and 93 control women. We defined the immune profile as the presence of 2 or more of the following abnormalities: Peripheral blood NK cell percentages $>15 \%$, positive antiphospholipid antibodies, positive antinuclear antibodies, positive anti-thyroid antibodies, low complement C3 levels and low C4 complement levels. Evolution to autoimmune diseases was detected during follow-up. Lymphocyte subsets were evaluated by flow-cytometry. Statistics: Chi-square test. Logistic regression.

Results: The prevalence of women with 2 or more immunological abnormalities was 57 out of 366 women (15.6\%) and was significantly higher than in control women. Demographic clinical characteristics were similar in women with 2 or more immunological abnormalities as compared with women with only one immunological alteration or no abnormalities. The presence of the immunological profile was significantly associated with the presence of the following clinical characteristics: Leucopenia $(p=0.048)$, lymphopenia $(p=0.007)$, livedo reticularis $(p=0.01)$, cutaneous rash $(p=0.009)$, and arthritis $(p=0.001)$. During follow-up 17 patients $(4.6 \%)$ developed an inflammatory or autoimmune disease that was not present at the time of the diagnose of RPL including SLE and lupus like disease. Women with the immunological profile were at higher risk for evolution into these diseases: OR 4.19, 95\% confidence interval $1.52-11.51, p=0.0055$. In 10 women with the immunological profile we observed significantly higher levels of CD4+DR+ and CD8+DR+ T-cells as compared with women without the immune profile.
Conclusions: A subgroup of women with unexplained RPL are at risk of developing clinical characteristics of an inflammatory or autoimmune disease. In this regard, the immunological evaluation of women with RPL might be necessary not only to identify a potential cause of abortion but also to identify women that could require a more careful clinical follow-up. Higher CD4+DR+ and CD8+DR+ T-cells might be a pathogenic pathway leading to development of autoimmune diseases in RPL women.

References:

[1] Viallard JF, Bloch-Michel C, Neau-Cransac M, Taupin JL, Garrigue S, Miossec V, Mercie P, Pellegrin JL, Moreau JF. HLA-DR expression on lymphocyte subsets as a marker of disease activity in patients with systemic lupus erythematosus. Clin Exp Immunol. 2001;125(3):485-91.

[2] CD8+DR+ T-Cells and C3 Complement Serum Concentration as Potential Biomarkers in Thrombotic Antiphospholipid Syndrome. Sarmiento E, Dale J, Arraya M, Gallego A, Lanio N, Navarro J, Carbone J. Autoimmune Dis. 2014. Acknowledgements: Research Funds. Fundacion Salud 2000. Madrid Spain. Disclosure of Interest: None declared

DOI: 10.1136/annrheumdis-2017-eular.6003

\section{FRI0284 PREDICTORS OF ARTERIAL VASCULAR EVENTS IN A COHORT OF SYSTEMIC LUPUS ERYTHEMATOSUS}

J.-G. Erdozain ${ }^{1}$, I. Villar ${ }^{2}$, J. Nieto ${ }^{2}$, I. Ruiz-Arruza ${ }^{1}$, J.-I. Pijoan ${ }^{3}$, G. Ruiz-Irastorza ${ }^{1} .{ }^{1}$ Unidad de Investigacion de Enfermedades Autoinmunes-Servicio de Medicina Interna. BioCruces Health Research Institute. Universidad del País Vasco/Euskal Herriko Unibertsitatea; ${ }^{2}$ Servicio de Medicina Interna: ${ }^{3}$ Unidad de Investigación. Unidad de Epidemiología Clínica y Soporte Metodológico. BioCruces Health Research Institute., Hospital Universitario de Cruces, Barakaldo-Vizcaya, Spain

Background: Arterial vascular events (AVE) are among the major causes of morbidity and mortality in patients with Systemic Lupus Erythematosus (SLE). Several studies have been carried out to identify the main factors related to AVE in this population. The ankle brachial index $(A B I)$ is one of the tools currently used to identify patients at greater risk of arterial events in the general population; however it has been scarcely studied in patients with SLE.

Objectives: The objectives of this prospective cohort study were to determine the predictive value of the ABI for occurrence of AVE in patients with SLE and to identify other possible factors associated with an increased risk of AVE.

Methods: 216 patients with SLE were evaluated using an $A B I$ and followed up for 5 years. Pathological $A B I$ is considered an $A B I<0.9$. Different potential vascular risk factors (traditional, non-traditional and related to SLE and/or the treatments used) were jointly evaluated. AVE: coronary events (angina pectoris, acute myocardial infarction, coronary revascularization by angioplasty or surgery), cerebrovascular events (transient ischemic attack, cerebrovascular accident), peripheral arterial disease (symptomatic intermittent claudication, distal ischemia, revascularization by angioplasty or surgery), and death related to vascular disease. Survival analysis was performed using a competitive risk regression approach, considering non-vascular death as a competitive event, to identify the predictive value of $\mathrm{ABI}$ and other factors studied. The Ethical Committee for Clinical Research at Cruces University Hospital approved the study protocol in accordance with the Declaration of Helsinki (CEIC E09/07). All patients signed an informed consent at the time of entry into the study.

Results: During follow-up, 4/216 (1.8\%) patients were lost to follow-up. 18 AVE were identified in 17 patients, with one patient having 2 episodes of angina requiring angioplasty ( 4 coronary events, 11 cerebrovascular events, 2 peripheral arterial disease events and 1 sudden death) and 14 deaths (6 per AVE or their sequelae, 4 due to neoplasias and 4 due to cardio-respiratory pathology). In the competitive risk regression analysis, independent predictors of higher risk of AVE were identified: pathological ABI (subhazard ratio (SHR) 3.51, 95\% confidence interval $0.96-12.79, p=0.057$ ), family history of AVE (SHR $6.3,95 \%$ Cl 1.97-20.21, $\mathrm{p}=0.002$ ), cumulative total prednisone (grams) (SHR 1.02, 95\% $\mathrm{Cl} 1.01-1.04, p=0.004$ ) and a history of arterial thrombosis (SHR $4.60,95 \% \mathrm{Cl}$ $1.45-14,59, \mathrm{p}=0.010)$. Female gender was a protective factor for the occurrence of AVE (SHR $0.12,95 \% \mathrm{Cl} 0.04-0.40, p<0.0001$ ).

Conclusions: Being male, having a higher cumulative dose of prednisone, having a family history of early vascular disease and having suffered previous arterial thrombosis are independent risk predictors of an AVE in patients with SLE. Having abnormal ABI, even without statistical significance, showed a marked tendency to increase this risk despite the low number of events recorded in the studied cohort. Disclosure of Interest: None declared

DOI: 10.1136/annrheumdis-2017-eular.3013

\section{FRI0285 LUPUS NEPHRITIS IS ASSOCIATED WITH INCREASED RATES OF HOSPITALIZATION AND IN-HOSPITAL MORTALITY COMPARED WITH NON-RENAL LUPUS AND MATCHED CONTROLS: AN ANALYSIS OF INSURANCE CLAIMS DATA}

K.A. Belendiuk, H. Trinh, M.D. Cascino, L. Dragone, D. Keebler, J. Garg. Genentech, Inc., South San Francisco, CA, United States

Background: Systemic lupus erythematosus (SLE) is heterogeneous in its clinical presentation, course, and prognosis and lupus nephritis (LN) continues 